\title{
Extra dimensions at the CERN LHC via mini-black holes: Effective Kerr-Newman brane-world effects
}

\author{
R. da Rocha* \\ Instituto de Física Teórica, Universidade Estadual Paulista, Rua Pamplona 145, 01405-900 São Paulo, SP, Brazil, \\ and DRCC-Instituto de Física Gleb Wataghin, Universidade Estadual de Campinas, CP 6165, 13083-970 Campinas, SP, Brazil \\ C. H. Coimbra-Araújo ${ }^{\dagger}$ \\ Instituto de Física Gleb Wataghin, Universidade Estadual de Campinas, CP 6165, 13083-970 Campinas, SP, Brazil
}

(Received 3 July 2006; published 22 September 2006)

\begin{abstract}
We solve Einstein equations on the brane to derive the exact form of the brane-world-corrected perturbations in Kerr-Newman singularities, using Randall-Sundrum and Arkani-Hamed-DimopoulosDvali (ADD) models. It is a consequence of such models that Kerr-Newman mini-black holes can be produced in LHC. We use this approach to derive a normalized correction for the Schwarzschild MyersPerry radius of a static $(4+n)$-dimensional mini-black hole, using more realistic approaches arising from Kerr-Newman mini-black hole analysis. Besides, we prove that there are four Kerr-Newman black hole horizons in the brane-world scenario we use, although only the outer horizon is relevant in the physical measurable processes. Parton cross sections in LHC and Hawking temperature are also investigated as functions of Planck mass (in the LHC range 1-10 TeV), mini-black hole mass, and the number of large extra dimensions in brane-world large extra-dimensional scenarios. In this case a more realistic braneeffect-corrected formalism can achieve more precisely the effective extra-dimensional Planck mass and the number of large extra dimensions - in the Arkani-Hamed-Dimopoulos-Dvali model—or the size of the warped extra dimension - in Randall-Sundrum formalism.
\end{abstract}

DOI: 10.1103/PhysRevD.74.055006

PACS numbers: 04.50.+h, 11.25.-w, 98.80.Jk

\section{INTRODUCTION}

The hierarchy problem, concerning the large ratio of the weak scale mass and the Planck scale mass, can possibly shed new light on the existence of large extra dimensions and, in order to improve some theories that have not been tested yet, their predictions - like compactification of extra dimensions of the order of the Planck length $\left(\approx 10^{-33} \mathrm{~cm}\right.$ ) - there have recently been proposed new approaches [1-5] suggesting that extra dimensions can be as large as around a millimeter. One of these approaches, the Arkani-Hamed-Dimopoulos-Dvali (ADD) formalism, essentially suggests a higher-dimensional compact manifold to describe the Universe, and explains how large extra dimensions could dilute the strength of gravity [4]. Although the gravitational force would be very strong in higher-dimensional spacetime, in the lower-dimensional effective theory gravity is weaker when the volume of the extra-dimensional compactified space is bigger. ADD predicted that this dilution of gravity into extra dimensions could possibly be so large that it can explain why the observed 4-dimensional gravity is so feeble. ADD models all have necessarily more than one extra curled up dimension - in order not to imply deviations in Newtonian gravity over solar system distances - wherein a single brane accommodates standard model particles. ADD models propose that the fundamental mass scale determining grav-

\footnotetext{
*Electronic address: roldao@ifi.unicamp.br

†Electronic address: carlosc@ifi.unicamp.br
}

ity strength is close to a TeV, instead of the Planck scale mass. It represents the fundamental higher-dimensional Planck scale.

Another formalism concerning large extra dimensions is the Randall-Sundrum brane-world model [2,3]. This formalism is effectively 5-dimensional, with one warped extra dimension, and it is based on a 5-dimensional reduction of $(1+9)$-dimensional brane Horava-Witten theory [6-9]. The idea that the Universe is trapped on a membrane in some higher-dimensional spacetime may explain why gravity is so weak, and could be tested at high energy particle accelerators. In this context, the observable universe proposed by Randall and Sundrum, in one of their two models, can be described as a brane embedded in an $\mathrm{AdS}_{5}$ bulk. At low energies gravity is localized on the brane and general relativity is recovered, but, at high energies, significant changes are introduced in gravitational dynamics, forcing general relativity to break down and to be overcome by a quantum gravity theory $[10,11]$. A plausible reason for the gravitational force to appear to be so weak can be its dilution in possibly existing extra dimensions related to a bulk, where $p$-branes [12-17] are embedded. $p$-branes are good candidates for brane worlds because they possess gauge symmetries [13-15] and automatically incorporate a quantum theory of gravity.

In Randall-Sundrum formalism gravity is also diluted in warped extra-dimensional space, but this time due to graviton's probability function (GPF) that is dependent on the distance to the gravity brane of the theory. While in ADD models the GPF is equally distributed over all the extension 
of extra-dimensional space, and 4-dimensional gravity becomes diluted, in Randall-Sundrum formalism GPF varies due to the warped geometry. By the effective warp factor in Randall-Sundrum formalism, all fundamental masses, expected to be of the order of $M_{\mathrm{P}}$ (the Planck scale mass) on an auxiliary (gravity) brane, become $10^{16}$ smaller, about a $\mathrm{TeV}$ magnitude. All masses are rescaled by the warp factor, and the 5-dimensional Planck scale is $10^{16} \mathrm{TeV}$, whereas the $M_{\mathrm{P}}$ denotes now the effective warp factor suppressed Planck scale.

In previous papers we have explored Randall-Sundrum (RS) formalism in the context of supermassive black holes (BHs) present in the nucleus of galaxies and quasars, and when they cause deviations from the 4D general relativity, these corrections should cause a small deviation in all $\mathrm{BH}$ properties $[18,19]$. It has already proposed the observation of brane-world BHs, and there are also other proposals on probing extra dimensions $[20,21]$. Now we want to investigate these brane-world effects for the case of mini-BHs and their possible measurable consequences in Large Hadron Collider (LHC) observations [22]. It is claimed that if the fundamental Planck scale is a TeV scale, LHC will produce over $10^{7}$ black holes per year [23].

Mini-BHs have radii that are much smaller than the size of extra dimensions, and therefore can be considered as totally embedded in a $D$-dimensional space. It is argued that, as particles approach each other in LHC, their gravitational attraction increases steadily, and they can enter extra-dimensional space when they are extremely close. In that case it would allow gravity to increase and a mini-BH could form. The mass of a mini-BH can be no smaller than Planck mass, of order 22 micrograms, corresponding to the mini-BH radius of the quantum fuzzy foam $\left(10^{-33} \mathrm{~m}\right)$. Also, mini-BH lifetime can be about $10^{-26} \mathrm{~s}$ and its temperature, typically around $80 \mathrm{GeV} \approx 1.5 \times 10^{14} \mathrm{~K}$, is much lower than it would be in a 4-dimensional spacetime, but still presents a high surface temperature though, at which mini-BHs would evaporate very rapidly into photons, electrons, and quarks, with energies ranging from $80 \mathrm{GeV}$ down. It is believed that mini-BHs would either evaporate completely or leave a remnant, and lifetime estimates were obtained in [24]. Although the standard model does not put a constraint on the minimum size $\mathrm{BH}$ production by an accelerator, of about less than $10^{16} \mathrm{TeV}$, some astounding ideas suggest that gravity becomes stronger at small distances because of the effects of extra dimensions accessed only by gravity.

First of all, the reconstruction of temperature as a function of mini-BH mass provides knowledge concerning the extra dimensions of spacetime $[23,25,26]$. In the case of Planck scales close to $\mathrm{TeV}$, the number of extra dimensions could thus be easily unraveled by the features of emitted particles. The mini-BHs production in LHC $[27,28]$ is an expected possibility and, as it is claimed, this will be the dominant effect in LHC under a quantum gravity theory
[29-31]. Also, a complete characterization of the Hawking radiation in extra-dimensional scenarios, showing that graviton emission becomes relevant for a large number of extra dimensions, can be seen in [32-36]. Exact stationary axisymmetric solutions describing rotating $\mathrm{BHs}$ - with tidal charge as well as with electric charge-localized on the brane in RS brane-world model have been investigated in [37]. Also, new solutions of the Einstein-Maxwell equations that describe an electrically charged and slowly rotating $\mathrm{BH}$ in five dimensions were discussed in [38-40].

We consider both Randall-Sundrum and ADD mini-BH production, because they present different character, as pointed out by Stojkovic [36]. While ADD mini-BHs have the first phase Hawking radiation mostly present in the bulk (and the second phase is mostly on the brane), existence of relaxation time during which the black hole looses the bulk components of angular momentum, and recoil effect to leave the brane, a Randall-Sundrum mini$\mathrm{BH}$ have bulk radiation strongly suppressed, do not have any bulk components of angular momentum (absence of relaxation time) and cannot recoil and leave the brane.

The main aim of this paper is to show how to correct, considering brane-world effects in ADD and RandallSundrum models, the mini-BH Kerr-Newman radii (horizons), by solving Einstein equations on the brane. In the case we consider, when the radius of a $\mathrm{BH}$ on the brane is much smaller than the size scale of the extra dimensions, the $\mathrm{BH}$ can be well described by the classical solutions of higher-dimensional Einstein equations. The Schwarzschild radius, in the context of Myers-Perry extra-dimensional formalism, is shown here to be significantly increased by tidal charge and spinning effects. The corrections we obtain give rise to more precise calculations concerning cross sections, Planck and mini-BHs masses, and Hawking temperature, contributing in this way to a more complete, precise, and realistic analysis of mini-BH production in the next generation of particle colliders, such as LHC.

This article is organized as follows: in Sec. II Einstein field equations on the brane are presented together with the brane-corrected Newtonian potential. Reissner-Nordstrøm $\mathrm{BHs}$ are also considered in a brane-world scenario viewpoint. In Sec. III by inputting a radial coordinate-valued function to perturb a Kerr-Newman singularity, we solve Einstein field equations on the brane to find the explicit form of these perturbations. Various graphics are depicted to illustrate the corrections in Kerr-Newman radii as functions of the BH angular momentum, both in RandallSundrum and ADD brane-world scenarios, for various fixed values of the $\mathrm{BH}$ charge. Also, cross sections are now corrected by brane-world effects and it gives rise to more realistic analysis of LHC collision products, such as mini- $\mathrm{BH}$ and particle production via mini-BH decay. The corrections in Kerr-Newman mini-BH radii are analyzed separately in a Randall-Sundrum brane-world scenariowhere there is $n=1$ warped extra dimension only - and in 
the ADD scenario with $n=2,4$, and 6 extra dimensions, as functions of Planck masses in the range $1-10 \mathrm{TeV}$, as well as $\mathrm{BH}$ masses and the $\mathrm{BH}$ spinning parameter. Graphics of parton cross sections are also shown as functions of Planck masses in the range $1-10 \mathrm{TeV}$ and $\mathrm{BH}$ masses. Finally, in Sec. IV, Kerr-Newman mini-BH Hawking evaporation and temperature are investigated in a brane-world scenario. All graphics present our results in explicit comparison with the Schwarzschild BH described by Myers-Perry standard results—-see, e.g., [23,25,26].

\section{MINI-BLACK HOLES ON THE BRANE}

In a brane-world scenario the Einstein field equations read $[18,19,41,42]$

$$
\begin{aligned}
G_{\mu \nu}= & -\frac{1}{2} \Lambda_{5} g_{\mu \nu}+\frac{1}{4} \kappa_{5}^{4}\left[T T_{\mu \nu}-T_{\nu}{ }^{\alpha} T_{\mu \alpha}\right. \\
& \left.+\frac{1}{2} g_{\mu \nu}\left(T^{2}-T_{\alpha \beta}{ }^{\alpha \beta}\right)\right]-E_{\mu \nu},
\end{aligned}
$$

where $T=T_{\alpha}{ }^{\alpha}$ denotes the trace of the energy-momentum tensor, $\Lambda_{5}$ denotes the 5-dimensional cosmological bulk constant, and $E_{\mu \nu}$ denotes the "electric" components of the Weyl tensor, which can be expressed by means of the extrinsic curvature components $K_{\mu \nu}=-\frac{1}{2} \mathcal{L}_{n} g_{\mu \nu}$ by [43]

$$
E_{\mu \nu}=\mathcal{L}_{n} K_{\mu \nu}+K_{\mu}{ }^{\alpha} K_{\alpha \nu}-\frac{1}{\ell^{2}} g_{\mu \nu},
$$

where $\ell$ denotes the bulk curvature radius. It corresponds equivalently to the effective size of the extra dimension probed by a $5 \mathrm{D}$ graviton [2,3,42]. Let $\kappa_{5}$ be defined as $\kappa_{5}=8 \pi G_{5}$, where $G_{5}$ denotes the 5-dimensional Newton gravitational constant, which can be related to the 4dimensional gravitational constant $G$ by $G_{5}=G \ell_{\text {Planck }}$, where $\ell_{\text {Planck }}=\sqrt{G \hbar / c^{3}}$ is the Planck length.

As indicated in [3,42], “...tabletop tests of Newton's law currently find no deviations down to the order of $0.1 \mathrm{~mm}$...," so that $\ell \leqslant 0.1 \mathrm{~mm}[30,44]$ provides a more accurate magnitude limit improvement on the bulk curvature $\ell$. The Weyl "electric" term $E_{\mu \nu}$ carries an imprint of high-energy effects sourcing Kaluza-Klein (KK) modes. The component $E_{\mu \nu}$ also carries information about the collapse process of BHs. In the perturbative analysis of the Randall-Sundrum positive tension 3-brane, KK modes consist of a continuous spectrum without any gap [45]. It generates a correction in the gravitational potential $V(r)=$ $\frac{G M}{c^{2} r}$ to $4 \mathrm{D}$ gravity at low energies by extra-dimensional effects [42], which is given by [2,3], for $r \gg \ell$

$$
V(r)=\frac{G M}{c^{2} r}\left[1+\frac{2 \ell^{2}}{3 r^{2}}+\mathcal{O}\left(\frac{\ell}{r}\right)^{4}\right],
$$

and for $r \ll \ell$,

$$
V(r) \approx \frac{G M \ell}{c^{2} r^{2}} .
$$

The KK modes that generate these corrections are respon- sible for a nonzero $E_{\mu \nu}$. This term carries the modification to the weak-field equations, as we have already seen.

For a static spherical metric on the brane given by

$$
g_{\mu \nu} d x^{\mu} d x^{\nu}=-F(r) d t^{2}+\frac{d r^{2}}{H(r)}+r^{2} d \Omega^{2},
$$

the projected electric component Weyl term on the brane is given by the expressions $[18,19,42]$

$$
\begin{aligned}
& E_{00}=\frac{F}{r}\left(H^{\prime}-\frac{1-H}{r}\right), \\
& E_{r r}=-\frac{1}{r H}\left(\frac{F^{\prime}}{F}-\frac{1-H}{r}\right), \\
& E_{\theta \theta}=-1+H+\frac{r}{2} H\left(\frac{F^{\prime}}{F}+\frac{H^{\prime}}{H}\right) .
\end{aligned}
$$

Note that in Eq. (4) the Schwarzschild metric is recovered if $F(r)$ equals $H(r)$. It is well known that the most general solutions of Eq. (4), in the case of a Reissner-Nordstrøm $(\mathrm{RN}) \mathrm{BH}$, are given by $[42,46]$

$$
F(r)=1-\frac{2 G M}{c^{2} r}-\psi(r)=1-\frac{2 G M}{c^{2} r}+\frac{2 G \ell Q^{*}}{c^{2} r^{2}},
$$

where $M$ and $Q^{*}$ denote, respectively, the mass and the tidal charge induced by the bulk of an effective RN BH. The bulk induces a tidal charge $\mathrm{BH}$ on the brane. The radial coordinate-valued $\psi(r)$ is obtained when one substitutes $F(r)$ in Einstein equations on the brane [see Eq. (13)]. We use in the next section an analogous procedure to obtain the brane-world form of Kerr-Newman metric.

As asserted in [42], a negative $Q^{*}$ strengthens the gravitational field, since it arises from the source mass $M$ on the brane. By contrast, in the RN solution of general relativity, $Q^{*} \sim q^{2}$, where $q$ denotes the RN mini-BH electric and tidal charges, and this weakens the gravitational field. Negative tidal charge also preserves the spacelike nature of the singularity. The tidal-charge $\mathrm{BH}$ metric does not satisfy the far-field $r^{-3}$ correction to the gravitational potential, however Eq. (6) shows the correct 5D behavior of the potential at short distances, so that the tidal-charge metric could be a good approximation in the strong-field regime for small BHs [42], as seen in Eq. (3). Equations (3) and (6) also imply, for extremal BHs defined by the relation

$$
Q^{*}=-2 M,
$$

that RN BHs in brane-world models also have two-inner and outer-horizons, denoted by $R_{\mathrm{RNbrane}}^{ \pm}$, which can be obtained by fixing $F(r)=0$ in Eq. (6). It results in [42]

$$
R_{\mathrm{RNbrane}}^{ \pm}=\frac{G M}{c^{2}} \pm \frac{1}{c}\left[\frac{G^{2} M^{2}}{c^{2}}-2 \ell G Q^{*}\right]^{1 / 2} .
$$

There are two horizons given by Eq. (8) and the charge $Q^{*}$ is constrained by the real-valued square root in the same equation. In the next section, we will see that, in a braneworld scenario Kerr-Newman BHs presents four horizons. 


\section{REALISTIC KERR-NEWMAN MINI-BHS AT LHC}

Our assumption is now to consider a realistic mini-BH to be produced in LHC, which is unlikely static, and the realistic conception is to proceed by considering a spinning, charged Kerr-Newman mini-BH. It has been demonstrated [47] that half a mini-BH mass is emitted when it is highly rotating, confirming it is of primordial importance to take into account the angular momentum of BHs. The Kerr-Newman metric, in Boyer-Lindquist coordinates, describing the neighborhood of a spherical rotating $\mathrm{BH}$ with mass $M$, angular momentum $J$, and charge $Q$, is given by

$$
g_{\mu \nu}^{\mathrm{K}-\mathrm{N}}=\left(\begin{array}{cccc}
\gamma / \rho^{2} & 0 & 0 & -\omega \beta^{2}+\alpha \\
0 & \rho^{2} / \Delta & 0 & 0 \\
0 & 0 & \rho^{2} & 0 \\
-\omega \beta^{2}+\alpha & 0 & 0 & \beta^{2}
\end{array}\right),
$$

where

$$
\begin{gathered}
\Delta=r^{2}+\frac{a^{2}}{c^{2}}-2 \frac{G M}{c^{2}} r+Q^{2}, \quad \rho^{2}=r^{2}+\frac{a^{2}}{c^{2}} \cos ^{2} \theta, \\
\Sigma^{2}=\left(r^{2}+\frac{a^{2}}{c^{2}}\right)^{2}-\frac{a^{2}}{c^{2}} \Delta \sin ^{2} \theta, \quad \beta=\frac{\Sigma}{\rho} \sin \theta, \\
\omega=\frac{2 a G M r}{c^{3} \Sigma^{2}}, \quad \alpha=\frac{a \sin ^{2} \theta Q^{2}}{c \rho^{2}}, \\
\gamma=\frac{a^{2} \sin ^{2} \theta}{c^{2}}-\Delta .
\end{gathered}
$$

The spinning parameter $a$ is defined as $a=\frac{J}{M c}$. In order to write the Kerr-Newman metric in a diagonal form, when we solve the characteristic eigenvalue equation associated with Eq. (9), the eigenvalues are given by

$$
\begin{gathered}
\lambda_{2}=\frac{\rho^{2}}{\Delta}, \quad \lambda_{3}=\rho^{2}, \\
\lambda_{1,4}=\frac{1}{2}\left[\gamma / \rho^{2}+\beta^{2} \pm\left(\gamma^{2} / \rho^{4}+4\left(\alpha-\omega \beta^{2}\right)\right.\right. \\
\left.\left.-2 \beta^{2} \gamma / \rho^{2}+\beta^{4}\right)^{1 / 2}\right] .
\end{gathered}
$$

Now we must impose a condition - arising when the eigenvalue characteristic equation is solved - for real eigenvalues, i.e.,

$$
\gamma^{2} / \rho^{4}+4\left(\alpha-\omega \beta^{2}\right)-2 \beta^{2} \gamma / \rho^{2}+\beta^{4} \geq 0,
$$

from which the Kerr-Newman metric is given in a diagonal form:

$$
\begin{aligned}
g^{\mathrm{K}-\mathrm{N}} & =g_{\mu \nu}^{\mathrm{K}-\mathrm{N}} d x^{\mu} d x^{\nu} \\
& =\lambda_{1} d t^{\prime 2}+\frac{d r^{2}}{\Delta / \rho^{2}}+\rho^{2} d \theta^{2}+\lambda^{4} d \phi^{\prime 2}
\end{aligned}
$$

Here $d \phi^{\prime}$ and $d t^{\prime}$ are 1-form fields on the 3-brane, respectively, related to $d \phi$ and $d t$ by the new eigenvectors in the associated directions defined by the eigenvalue equation associated with Eq. (9).

In order to obtain the correction of the Kerr-Newman radii, by brane-world effects, we follow the idea presented in Eq. (6). Besides, it is well known that a particular way to express the vacuum field equations on the brane is [42]

$$
E_{\mu \nu}=-R_{\mu \nu}, \quad R_{\mu}^{\mu}=0=E_{\mu}^{\mu}, \quad \nabla^{\nu} E_{\mu \nu}=0 .
$$

Defining $\xi(r)$ as the deviation from a Kerr-Newman form $\Delta / \rho^{2}$ - the term in the denominator of $d r^{2}$ in Eq. (12) we can now solve Eqs. (13), via Eqs. (5), for the particular case where $\mu=\nu=0$, obtaining

$$
\xi(r)=\frac{2 G \ell Q^{*}}{c^{2} r(r+a / c)}
$$

as one solution, in such a way that in a static limit $(a \rightarrow 0)$ the correction in the gravitational potential satisfies Eq. (3). Imposing that the charge in these mini-BHs are induced $a$ priori by bulk effects, we can define $Q=G Q^{*} / c^{2}$. Now, the corrections $R_{\mathrm{K} \text {-Nbrane }}$ in the Kerr-Newman radii are obtained via the deviated Kerr-Newman form, as

$$
\frac{\Delta}{\rho^{2}}+\xi\left(R_{\mathrm{K}-\mathrm{Nbrane}}\right)=0 .
$$

By expanding the expression above and using Eqs. (10) and (14) we obtain

$$
\begin{aligned}
R_{\mathrm{K} \text {-Nbrane }}^{4}+\Gamma_{3} R_{\mathrm{K} \text {-Nbrane }}^{3}+\Gamma_{2} R_{\mathrm{K}-\mathrm{Nbrane}}^{2}+ & \Gamma_{1} R_{\mathrm{K} \text {-Nbrane }} \\
& +\Gamma_{0}=0,
\end{aligned}
$$

where

$$
\begin{aligned}
& \Gamma_{3}=a / c-2 G M / c^{2}, \\
& \Gamma_{2}=a^{2} / c^{2}+2 \ell Q-2 a G M / c^{3}+Q^{2}, \\
& \Gamma_{1}=a^{3} / c^{3}+a Q^{2} / c, \\
& \Gamma_{0}=2 G \ell Q^{*} a^{2} \cos ^{2} \theta / c^{2} .
\end{aligned}
$$

A mini-BH not corrected by extra-dimensional tidalcharge effects can be simply described by a Kerr miniBH: the horizon radius comes from the expression obtained by making $Q=0$ in Eq. (12). Using natural units $(c=1$, $G=1$ ), by solving Eq. (16) we find results for the normalized brane-corrected Kerr-Newman radius $K(M, a, Q)$. In what follows, this normalized horizon will be used to make corrections to a conventional Schwarzschild radius $R_{S}$, in order to find a Kerr-Newman $\mathrm{BH}$ by $R_{\mathrm{K}-\mathrm{Nbrane}}=$ $\frac{1}{2} K(M, a, Q) R_{S}$. Equation (16) gives four solutions for $R_{\mathrm{K}-\mathrm{Nbrane}}$ and we depict below three graphics, respectively, representing the inner $R_{\mathrm{K}-\mathrm{Nbrane}}$ horizon corrections, intermediary $R_{\mathrm{K} \text {-Nbrane }}$ radius corrections, and the outer $R_{\mathrm{K}-\mathrm{Nbrane}}$ horizon corrections, as functions of the spinning parameter $a$, for specific values of the charge $Q^{*}$. Solutions for the normalized horizon $K(M, a, Q)$ with respect to the 


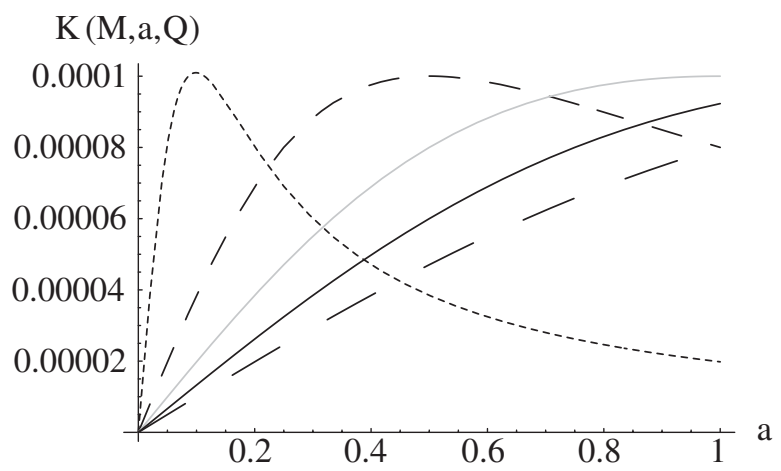

FIG. 1. Graphic of the brane-effect-corrected normalized inner horizon $K(M, a, Q) \times a$ for different values of $Q^{*}$. For the very short dashed line: $Q^{*}=-0.1$; for the short dashed line: $Q^{*}=$ -0.5 ; for the full gray line: $Q^{*}=-1$; for the full black line: $Q^{*}=-1.5$; and for the long dashed line: $Q^{*}=-2$.

spinning parameter factor $a$ are presented in Figs. 1-3, respectively, for inner, intermediate, and outer KerrNewman horizons.

These corrections can be applied to the Schwarzschild radius $R_{S}$ of a Myers-Perry $(4+n)$-dimensional $\mathrm{BH}$ $[23,29,48]$, resulting in

$$
\begin{aligned}
R_{\mathrm{K}-\mathrm{Nbrane}} & =\frac{1}{2} K(M, a, Q) R_{S} \\
& =\frac{K(M, a, Q)}{2 \sqrt{\pi} M_{\mathrm{P}(4+n)}}\left[\frac{M_{\mathrm{BH}}}{M_{\mathrm{P}(4+n)}}\left(\frac{8 \Gamma\left(\frac{n+3}{2}\right)}{n+2}\right)\right]^{1 /(n+1)}
\end{aligned}
$$

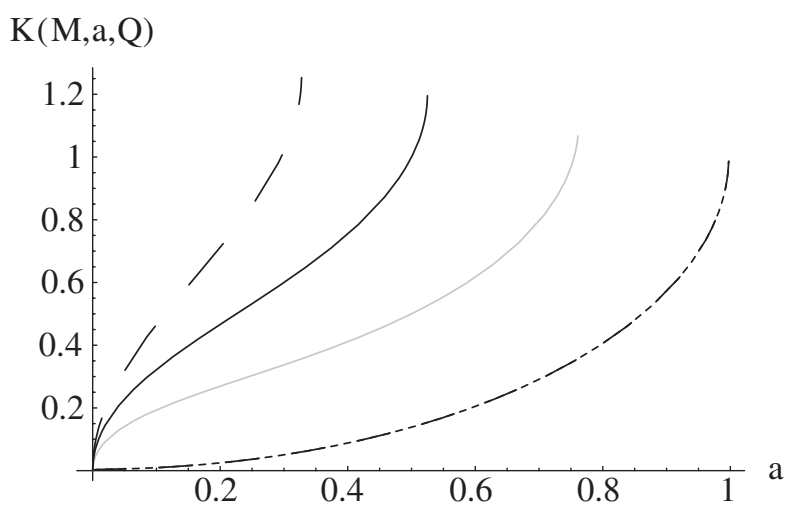

FIG. 2. Graphic of the brane-effect-corrected normalized horizon $K(M, a, Q) \times a$ for different values of $Q^{*}$. These are solutions for an intermediate horizon. For the very short dashed line: $Q^{*}=-0.1$; for the short dashed line: $Q^{*}=-0.5$; for the full gray line: $Q^{*}=-1$; for the full black line: $Q^{*}=-1.5$; and for the long dashed line: $Q^{*}=-2$. In this case, the spin of the mini$\mathrm{BH}$ is constrained by the electric and tidal charge. The maximum $a$ obtained for each graphic is: $a=0.32806$ for $Q^{*}=-2, a=$ 0.52572 for $Q^{*}=-1.5, a=0.76550$ for $Q^{*}=-1, a=$ 0.93842 for $Q^{*}=-0.5$, and $a=0.99753$ for $Q^{*}=-0.1$ (which practically coincides with the $Q^{*}=-0.5$ case). In these respective values for $a$, the derivative of $K(M, a, Q)$ related to $a$ tends to infinity.

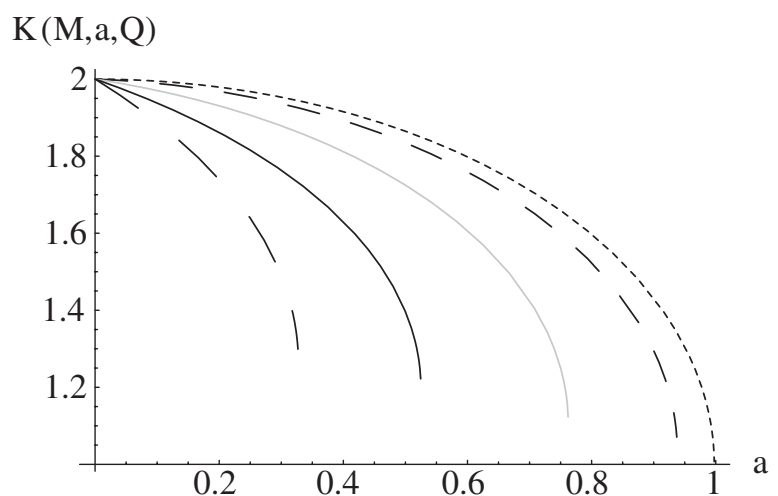

FIG. 3. Graphic of the brane-effect-corrected Kerr-Newman outer horizon in the form $c^{2} R_{\mathrm{K}-\mathrm{N}} / G M \times a$ for different values of $Q^{*}$. For the very short dashed line: $Q^{*}=-0.1$; for the short dashed line: $Q^{*}=-0.5$; for the full gray line: $Q^{*}=-1$; for the full black line: $Q^{*}=-1.5$; and for the long dashed line: $Q^{*}=$ -2 . In this case, the spin of the $\mathrm{BH}$ is constrained by the electric and tidal charge. The maximum $a$ obtained for each graphic is: $a=0.32791$ for $Q^{*}=-2, a=0.52766$ for $Q^{*}=-1.5, a=$ 0.76585 for $Q^{*}=-1, a=0.95826$ for $Q^{*}=-0.5$, and $a=$ 0.99866 for $Q^{*}=-0.1$.

where $\Gamma(m)$ denotes the usual Euler gamma function, $M_{\mathrm{P}(4+n)}$ is the $(4+n)$-dimensional Planck mass and $M_{\mathrm{BH}}$ is the mini-BH mass. Both $M_{\mathrm{P}(4+n)}$ and $M_{\mathrm{BH}}$ are given in $\mathrm{TeV}$. Expression (18) is normalized $(c=1, \hbar=1)$ and to obtain the value of $R_{\mathrm{K}-\mathrm{Nbrane}}$ in meters it is necessary to multiply the expression by $\hbar c=1.973 \times 10^{-13} \mathrm{MeV} \mathrm{m}$. The three solutions for $K(M, a, Q)$, represented by the graphics of Figs. 1-3 generate three horizons, which could be related to quantum $\mathrm{BH}$ theories [49]. Only the outer horizon expressed by Fig. 3 contributes to the total cross section (see Fig. 4).

Denoting by $M_{*}$ the strength of higher-dimensional gravity, $M_{\mathrm{P}} \approx 10^{16} \mathrm{TeV}$ sets the strength of 4-dimensional

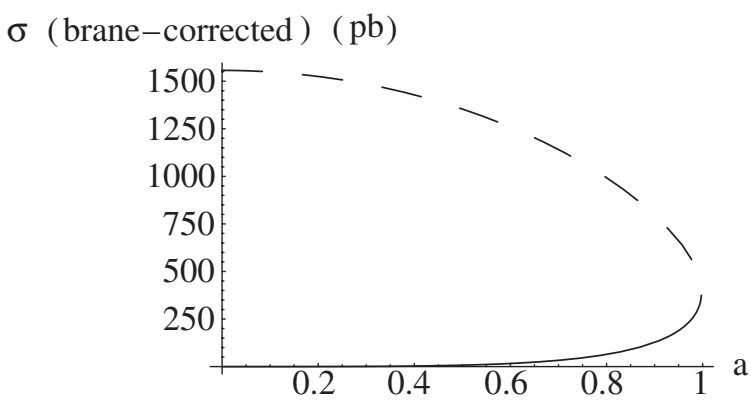

FIG. 4. Brane-effect-corrected Kerr-Newman cross section $\sigma$ for the inner horizon (full line) and for the outer horizon (dashed line) for various angular momenta $a$. Significant contributions by the inner horizon just happen when $a \rightarrow 1$. Thus, the effective contribution for the total cross section is only given by the outer horizon. This graphic presents an example for the $n=3$ extradimensional ADD model, $M_{\mathrm{P}}=1 \mathrm{TeV}, M_{\mathrm{BH}}=5 \mathrm{TeV}, Q^{*}=$ $-0.1 M_{\mathrm{BH}}$. 
gravity, and $V^{n}$ the volume of the higher-dimensional space, the 3-space-dimensional Newton's force law is recovered by the identification $[23,25,26,42]$

$$
M_{\mathrm{P}}^{2}=M_{*}^{n+2} V^{n} .
$$

Important differences concerning the number of extra dimensions in ADD and Randall-Sundrum formalism reside in the last expression. On the one hand, if there were only one large extra dimension in ADD model, gravity measured at, e.g., the solar system, would have a 5-dimensional nonobserved behavior. With $n=2$ extra dimensions, the size of extra dimensions would be a tenth of a millimeter. On the other hand, the Randall-Sundrum model is effectively 5-dimensional, with one extra warped dimension. In the graphics below we present our results for a certain number of extra dimensions, and it is implicit that, when we consider $n=1$ extra dimensions it is associated with Randall-Sundrum models, while for $n=2, \ldots, 6$ extra dimensions our results represent ADD models.

Considering two partons with the center of mass energy $\sqrt{\tilde{s}}=M$ moving in opposite directions, semiclassical reasoning suggests that the impact parameter is less than the Schwarzschild radius - in the case of an extremal Kerr-
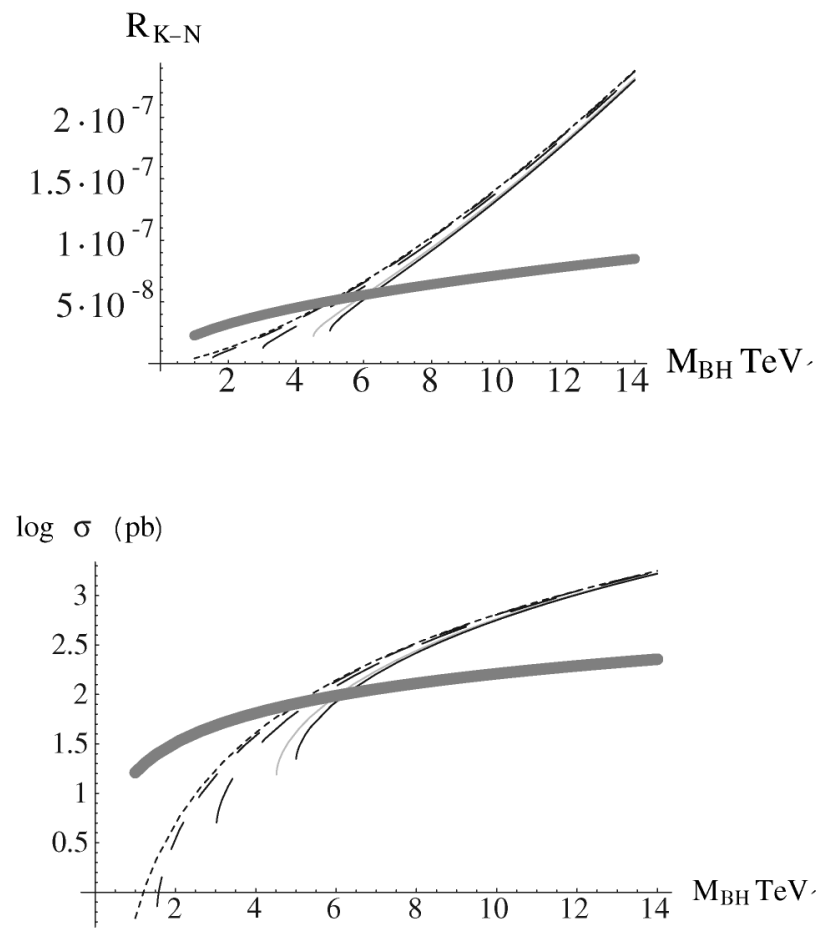

FIG. 5. Graphics of the brane-effect-corrected Kerr-Newman horizon $R_{\mathrm{K}-\mathrm{N}}$ of the produced mini- $\mathrm{BH}$ and its associated cross section, for various momenta $a$ versus $\mathrm{BH}$ masses, and its associated parton cross section for the Randall-Sundrum brane-world model, where there is one warped extra dimension. Here we present an example for $M_{\mathrm{P}}=4 \mathrm{TeV}$, and $Q^{*}=$ $-0.1 M_{\mathrm{BH}}$, with the short dashed line for $a=0.1$, the dashed line for $a=0.3$, the long dashed line for $a=0.6$, the gray full line for $a=0.9$, and the full black line for $a=0.997$.
Newman mini-BH - or less than the outer Kerr-Newman horizon-when the Kerr-Newman mini-BH is not extremal-a mini-BH with the mass $M$ arises. For the Schwarzschild BH particular case, see $[23,25,26]$. Parton distribution functions at LHC give the total cross section $\sigma$ for production of BHs with $M_{\mathrm{BH}}>M_{\mathrm{P}}$ in the range $1 \mathrm{pb}<$ $\sigma<15 \mathrm{pb}$, for $1 \mathrm{TeV}<M_{\mathrm{P}}<5 \mathrm{TeV}$ and it varies around $10 \%$ for $n$-the number of extra dimensions in ADD models - between 2 and 7 [25,26].

By elementary geometrical arguments, the total cross section is given by $[30,48]$

$$
\begin{aligned}
\sigma\left(M_{\mathrm{BH}}\right) & \approx \pi R_{\mathrm{K}-\mathrm{Nbrane}}^{2} \\
& =\frac{K^{2}(M, a, Q)}{4 M_{\mathrm{P}(4+n)}^{2}}\left[\frac{M_{\mathrm{BH}}}{M_{\mathrm{P}(4+n)}}\left(\frac{8 \Gamma\left(\frac{n+3}{2}\right)}{n+2}\right)\right]^{2 /(n+1)} .
\end{aligned}
$$

Now, in Figs. 5-9 we illustrate the brane effects in KerrNewman mini-BHs - for different values of charge - as functions of mini-BH mass, Planck mass, and spinning parameter (respectively in Randall-Sundrum and $n=2$, 4, and 6 extra-dimensional ADD models). In these graphics the wide gray line indicates the standard MyersPerry approach for Schwarzschild mini-BHs. We show in this way a more realistic and precise prediction concerning mini-BH production in LHC, since our approach does not neglect spin and charge effects.
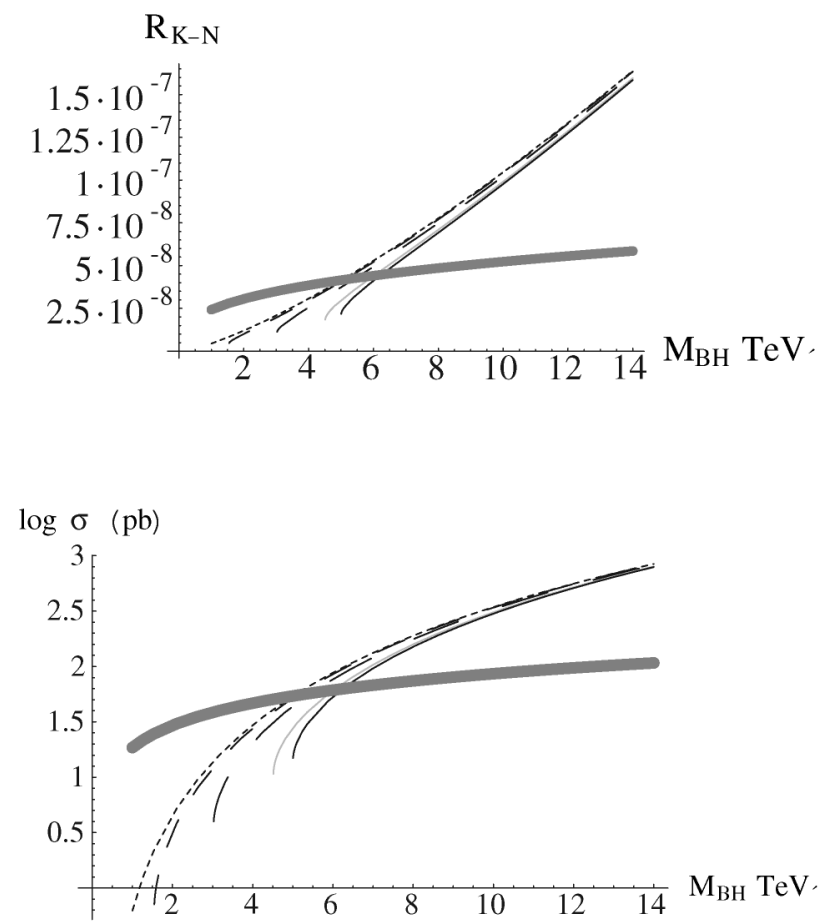

FIG. 6. Graphics of the brane-effect-corrected Kerr-Newman radius and its associated parton cross section for $n=2$ ADD model for some $\mathrm{BH}$ masses, with $M_{\mathrm{P}}=1 \mathrm{TeV}$, and for various angular momenta: short dashed line for $a=0.1$, dashed line for $a=0.3$, long dashed line for $a=0.6$, gray full line for $a=0.9$, and full black line for $a=0.997$. 
In Fig. 10 we show the parton cross section for the case of Schwarzschild mini-BH Myers-Perry production within the LHC range of masses, and in Fig. 11 we show the parton cross section for the case of production of a spinning charged mini-BH within the LHC range of masses in the brane-world Randall-Sundrum scenario. We present the same graphics for $n=2$ and $n=6$ extra dimensions, respectively, in Fig. 12, and in these cases, we obtain an

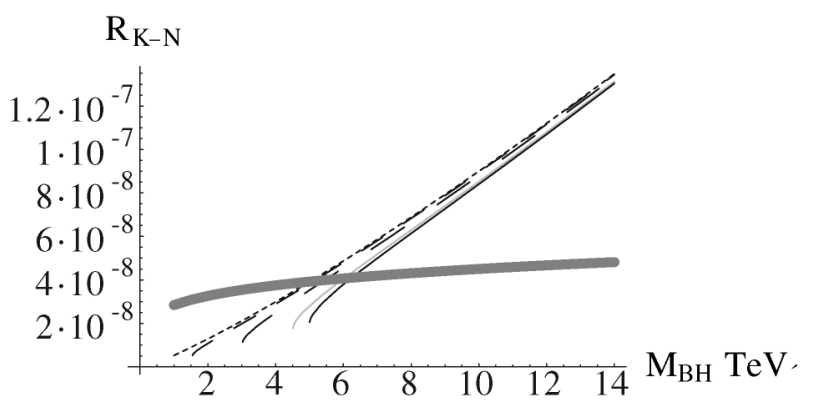

$\log \sigma(\mathrm{pb})$

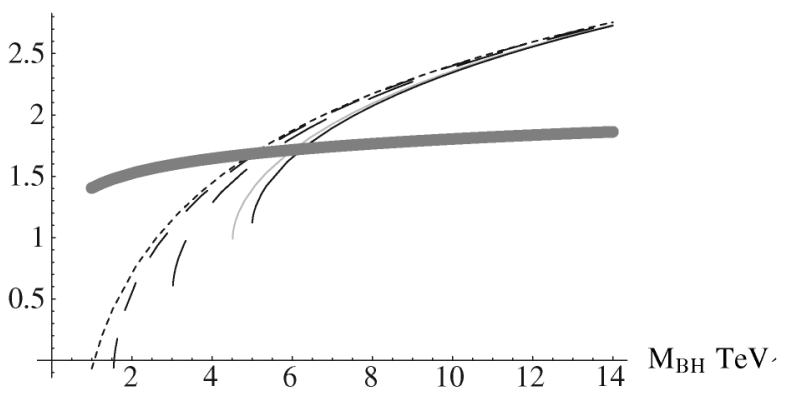

FIG. 7. Graphics of the brane-effect-corrected Kerr-Newman outer horizon and its associated parton cross section in the $n=4$ ADD model for some mini-BH masses, with $M_{\mathrm{P}}=1 \mathrm{TeV}$, and for various angular momenta: short dashed line for $a=0.1$, dashed line for $a=0.3$, long dashed line for $a=0.6$, gray full line for $a=0.9$, and full black line for $a=0.997$.

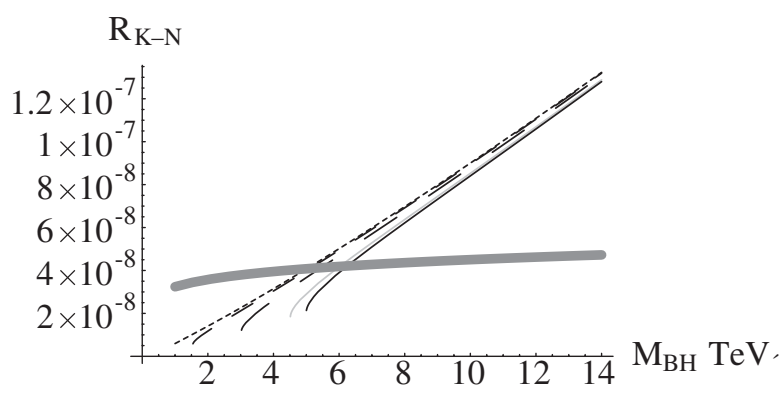

FIG. 8. Graphic of the brane-effect-corrected Kerr-Newman outer horizon for the extra-dimensional $n=6$ ADD model for some $\mathrm{BH}$ masses, with $M_{\mathrm{P}}=1 \mathrm{TeV}$, and for various angular momenta: short dashed line for $a=0.1$, dashed line for $a=0.3$, long dashed line for $a=0.6$, gray full line for $a=0.9$, and full black line for $a=0.997$. attenuation in the cross section values when the $\mathrm{BH}$ mass increases.

In Fig. 13 we show the parton cross section for the case of production of a spinning mini-BH within the LHC range

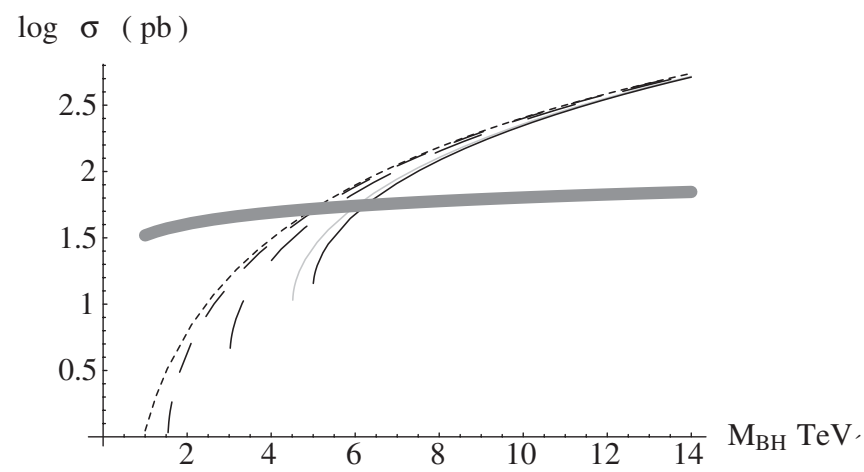

FIG. 9. Cross sections for $n=6$ extra dimensions in ADD model, short dashed line for $a=0.1$, dashed line for $a=0.3$, long dashed line for $a=0.6$, gray full line for $a=0.9$, and full black line for $a=0.997$.

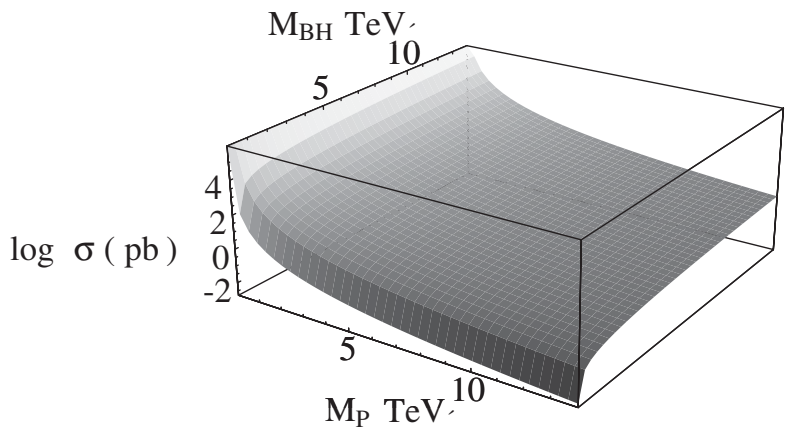

FIG. 10. Graphic of parton cross section in picobarn for various values of Planck mass $M_{\mathrm{P}}$ and $\mathrm{BH}$ mass $M_{\mathrm{BH}}$ for the Schwarzschild Myers-Perry model. The mini-BH formed by the scattering has $a=0.5$ and is charged $\left(Q^{*}=-1 M_{\mathrm{BH}}\right)$, for the Randall-Sundrum brane-world model.

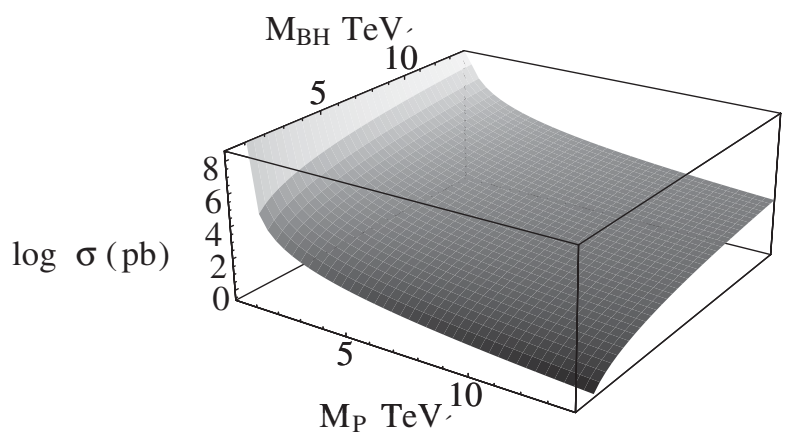

FIG. 11. Graphic of parton cross section in picobarn for various values of Planck mass $M_{\mathrm{P}}$ and $\mathrm{BH}$ mass $M_{\mathrm{BH}}$. The mini-BH formed by the scattering has $a=0.5$ and is charged with $Q^{*}=$ $-1 M_{\mathrm{BH}}$, for the Randall-Sundrum brane-world model. 

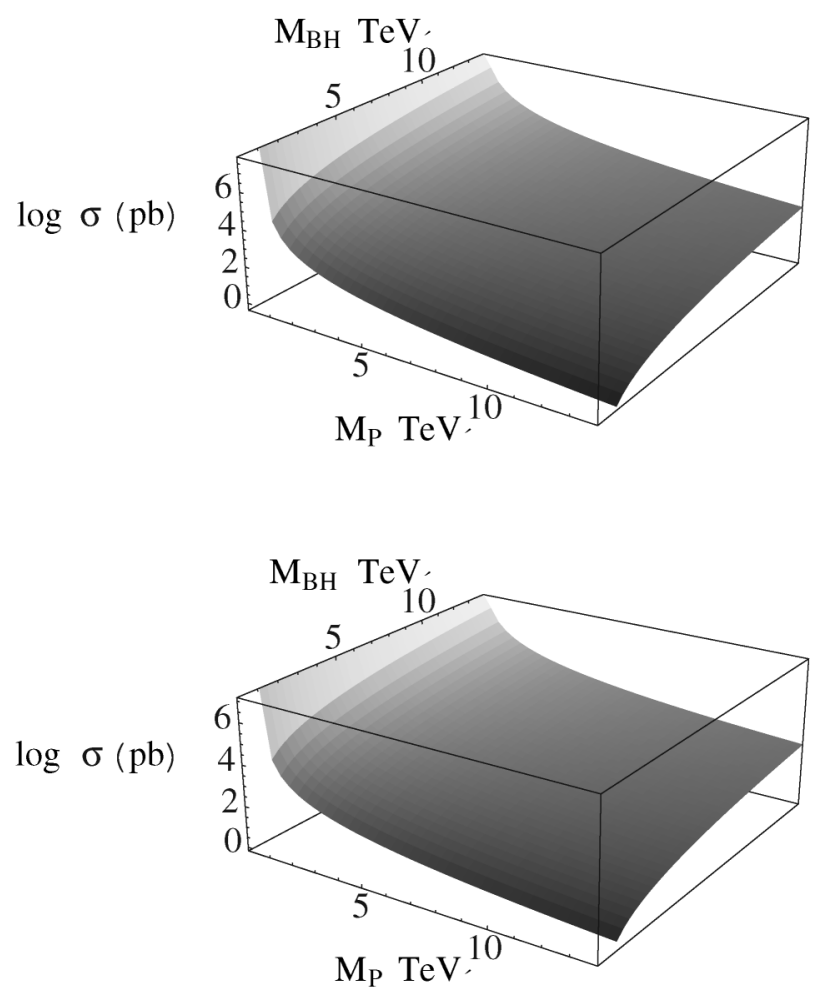

FIG. 12. Graphics of parton cross section in picobarn for various values of Planck mass $M_{\mathrm{P}}$ and $\mathrm{BH}$ mass $M_{\mathrm{BH}}$. The mini-BH formed by the scattering has $a=0.5$ and is charged with $Q^{*}=-1 M_{\mathrm{BH}}$, in respectively $n=2$ and $n=6$ extra dimensions in ADD model.

of masses, and for the case where the scenario is a RandallSundrum brane-world model; here we show explicitly the effect of the spinning parameter $a$. We present the same graphic for higher dimensions in Figs. 14 and 15. It is notorious the interesting effect caused by the spinning parameter $a$ in the cross section. The higher the number of extra dimensions, for instance $n=6$ as illustrated in Fig. 15, the faster the bending of the graphic is, as the spinning parameter $a \rightarrow 1$.

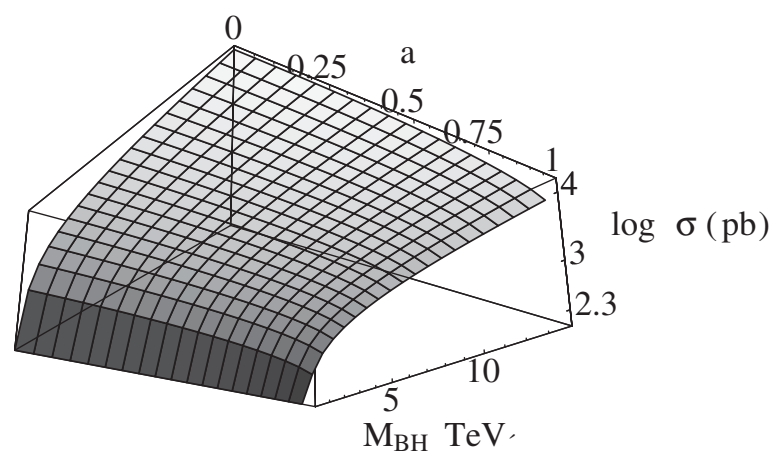

FIG. 13. Graphic of parton cross section in picobarn for various values of spinning parameter $a$ and $\mathrm{BH}$ mass $M_{\mathrm{BH}}$. The mini$\mathrm{BH}$ formed by the scattering is charged with $Q^{*}=-1 M_{\mathrm{BH}}$. In this case we consider the Randall-Sundrum brane-world model.

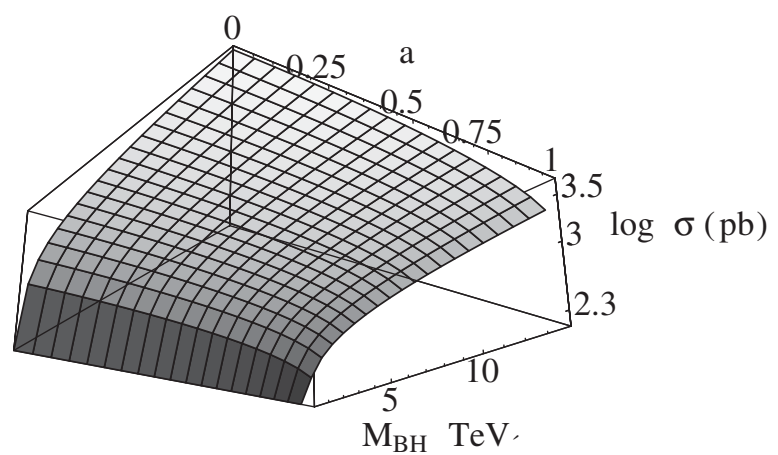

FIG. 14. Graphic of parton cross section in picobarn for various values of $a$ and the BH mass $M_{\mathrm{BH}}$. Note the interesting effect caused by the spinning parameter $a$ in the cross section. Here we consider the $n=2$ extra-dimensional ADD model, the mini-BH is electrically and tidally charged by the bulk with $Q^{*}=$ $-0.1 M_{\mathrm{BH}}$.

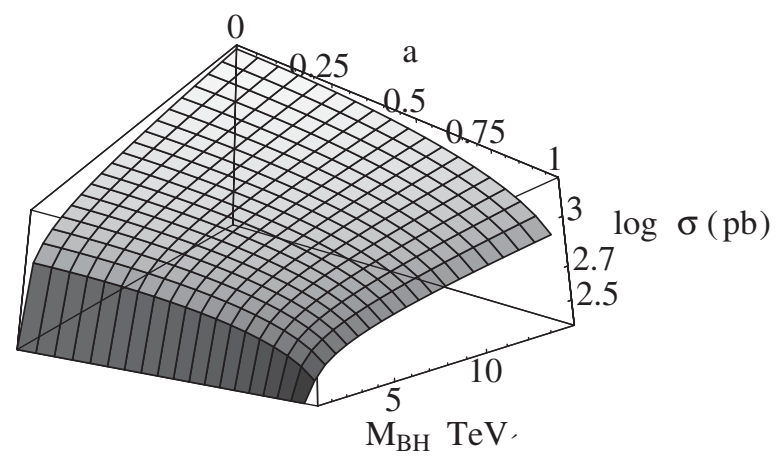

FIG. 15. Graphic of parton cross section in picobarn for various values of $a$ and the $\mathrm{BH}$ mass $M_{\mathrm{BH}}$. Note the interesting effect caused by the spinning parameter $a$ in the cross section. In this case, i.e., the $n=6$ extra-dimensional ADD model, the bending is more intense. The mini-BH has $Q^{*}=-0.1 M_{\mathrm{BH}}$.

\section{BH EVAPORATION AND HAWKING RADIATION IN KERR-NEWMAN MINI-BHS}

BHs can evaporate by emitting pairs of virtual photons at the event horizon, with one of the photon escaping the BH gravity [50], and have a blackbody spectrum with the Hawking temperature $\frac{\hbar c}{4 \pi k R_{S}}$. Hawking evaporation is significant only in the case of small BHs, where the tidal effect becomes so intense near the surface that the particle pairs produced by quantum vacuum fluctuations may be broken, one particle falling into the $\mathrm{BH}$ and the other being projected outwards.

$\mathrm{BH}$ production in $\mathrm{LHC}$ is expected immediately above the $M_{P}$ threshold, since at energies below $M_{P}$ other quantum effects overcome $\mathrm{BH}$ production. In $[25,26]$ it was shown how the relationship between $M_{\mathrm{BH}}$ and Hawking temperature can unravel Planck mass and the dimensionality $n$ of extra dimensions, independently of the geometry of extra dimensions. Although when a BH event horizon is observed by its Hawking radiation it looks like a fuzzy 
sphere (see [51]) — and in the classical limit the event horizon looks locally like a noncommutative plane with noncommutative parameter dictated by the Planck length-in particular at $M_{P}$ energies BHs are quantum objects and here we use as in [26] semiclassical arguments. Besides, mini-BHs produced at LHC would be light and extremely hot, with a Hawking temperature $T_{\mathrm{H}} \approx$ $100 \mathrm{GeV}$, and evaporate almost instantaneously, mainly via Hawking radiation.

Evaporation of a BH in $n$ large extra dimensions occurs at Hawking temperature $T_{\mathrm{H}}$, given in our model by

$$
T_{\mathrm{H}}=\frac{2 M_{P}}{K(M, a, Q)}\left(\frac{M_{\mathrm{BH}}}{M_{\mathrm{P}}} \frac{n+2}{8 \Gamma\left(\frac{n+3}{2}\right)}\right)^{1 /(n+1)} \frac{n+1}{4 \sqrt{\pi}} .
$$

We depict below $T_{\mathrm{H}}$ for different values of Kerr-Newman mini-BH charges.

In this case, as the charge $Q^{*}$ increases, the Hawking temperature increases as well. On the other hand, as the mini-BH mass increases, $T_{\mathrm{H}}$ decreases, and for all values of $Q^{*}$, Hawking temperature $T_{\mathrm{H}}$ tends to the same value as $M_{\mathrm{BH}}$ is $\sim 10 \mathrm{TeV}$.

It is notorious by the graphic above that as the spin parameter $a$ increases $T_{\mathrm{H}}$ increases as well.

In this case Hawking temperature is about $\sim 10^{1}-10^{2} \mathrm{GeV}$. The superior line indicates the graphic for a Schwarzschild BH Myers-Perry model $[23,25]$.

\section{CONCLUDING REMARKS AND OUTLOOKS}

By solving Einstein equations on the brane, we have found an explicit form for the perturbation of a KerrNewman metric singularity, corresponding to a KerrNewman BH. Such perturbation gives rise to more realistic calculations and predictions involving mini-BH production at LHC, since we concern brane-world corrections as they would appear in the measurement of cross sections, Hawking temperature and some other physical observations related to the mini-BH production at LHC. Mini-BHs are a natural choice for testing brane-world formalisms: the lighter the $\mathrm{BH}$, the greater its surface gravity, and consequently mini-BHs are more sensitive on brane-world physical effects, like, e.g., Hawking evaporation in the context of Randall-Sundrum and ADD models. Also, the $14 \mathrm{TeV}$ maximum energy of LHC could allow it to become a mini-BH factory with a production rate as high as about one per second, and after formation, mini-BHs are expected to lose the information associated with multipole and angular momenta, to decay via Hawking radiation, and eventually either disappear completely or leave a Plancksized remnant [23,52-54] around Planck masses $M_{\mathrm{P}}=$ $1.6 \mathrm{TeV}$ for $D=6$ and $M_{\mathrm{P}}=0.25 \mathrm{TeV}$ for $D=10$ [55].

Figures 1-3 in Sec. III show the corrections of the Schwarzschild radius as functions of the spin parameter $a$, for different values of the charge $Q^{*}$. Such brane-effect corrections arise immediately from the solutions of Einstein equations on the brane and they can describe more realistic predictions concerning mini-BHs features at LHC.

Brane-effect corrections for mini-BH cross sections at LHC are shown in Fig. 4 for $n=3$ extra dimensions in ADD model. Figures 5-9 also show the increment of the brane-effect-corrected Kerr-Newman BH horizons and the respective cross section as functions of the Kerr-Newman $\mathrm{BHs}$, for different values of charge, as functions of $\mathrm{BH}$ and Planck masses, and spinning parameter, respectively, for the Randall-Sundrum model and the $n=2, n=4$, and $n=6$ extra-dimensional ADD models. In all these graphics, the wide gray line indicates the standard Myers-Perry approach for Schwarzschild BHs, and it is clear that our results coming from Kerr-Newman analysis predict more accurately the results obtained from the static Schwarzschild Myers-Perry approach.

Figures 10-12 definitively express the brane corrections in mini-BH cross sections as functions of $\mathrm{BH}$ mass and the effective Planck mass in the range $1-10 \mathrm{TeV}$ of $\mathrm{LHC}$, respectively, for Schwarzschild-in Myers-Perry model - and Kerr-Newman mini-BHs for various values of extra dimensions both in Randall-Sundrum and ADD models. Figures 13-15 show the brane corrections in mini$\mathrm{BH}$ cross sections as functions of the spinning parameter $a$ and the mini-BH mass, in the range $1-10 \mathrm{TeV}$ of LHC, respectively, for various values of $n$ extra dimensions in Randall-Sundrum $(n=1)$, and ADD models (the particular cases where $n=2$ and $n=6$ ). Our results show prominent effects of a higher number of extra dimensions in cross sections and Hawking temperature associated with Kerr-Newman mini-BHs. Figure 16 illustrates the braneeffect-corrected cross sections as functions of $Q^{*}$ and the spinning parameter $a$, showing how the charge constrains the mini-BH spin.

Figures 17-19 illustrate Hawking temperature of a KerrNewman mini-BH, respectively, as a function of $\mathrm{BH}$ mass and spinning parameter $a$, both in an extra-dimensional scenario. Figure 18 shows the brane-world Kerr-Newman mini-BH corrections of the Schwarzschild Myers-Perry model. Again, for different values of mini-BH charge, $T_{\mathrm{H}}$

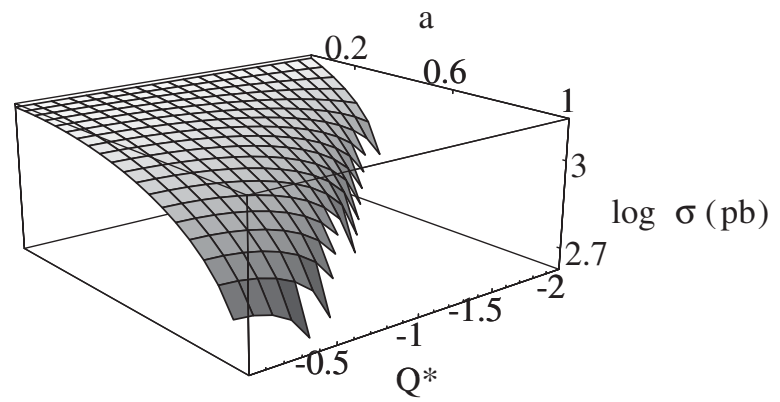

FIG. 16. Parton cross sections in picobarn for various values of $Q^{*}$ and $a$, explicitly showing the influence in the scattering if one considers a realistic Kerr-Newman mini-BH. The charge constrains the spin. 


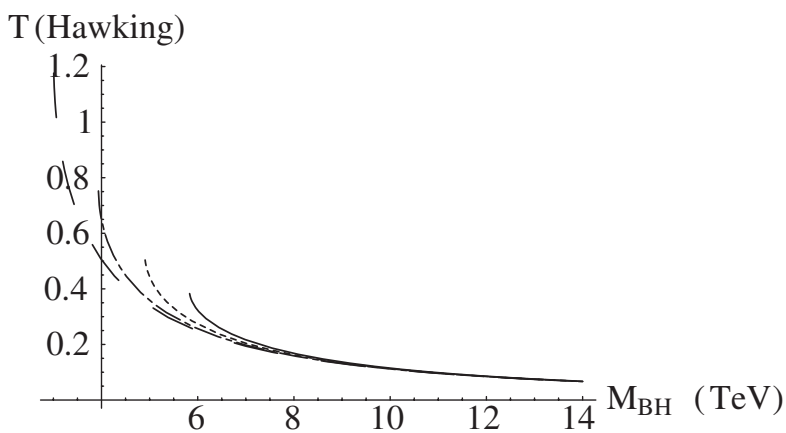

FIG. 17. Range of Hawking temperature, in $\mathrm{TeV}$, produced in LHC by a charged spinning $\mathrm{BH}$ evaporation versus $\mathrm{BH}$ mass interval. The range covers some values of charge for a fixed angular momentum: full line: $Q^{*}=-2 M$; dotted line: $Q^{*}=$ $-1.5 M$; dashed-dotted line : $Q^{*}=-M$; dashed line: $Q^{*}=$ $-0.5 M$, all for spinning parameter $a=0.5$. This means the $\mathrm{BH}$ charge is an important parameter in the determination of exact $\mathrm{BH}$ decay. Here, the Planck mass is $4 \mathrm{TeV}$ and $n=6$ for the Randall-Sundrum brane-world model.

seems not to be sensitive to these values for higher values of mini-BH mass, above $10 \mathrm{TeV}$. This shows that in the range $1-10 \mathrm{TeV}$ of LHC resides our brane-effect corrections. Also, from Figs. 17-19, as the charge $Q^{*}$ increases, the Hawking temperature increases as well. On the other hand, the greater the mini-BH mass, the lower the $T_{\mathrm{H}}$, and for all values of $Q^{*}$, Hawking temperature $T_{\mathrm{H}}$ tends to the same value as $M_{\mathrm{BH}}$ is $\sim 10 \mathrm{TeV}$. This allows us to follow $[25,26]$ in a brane-world viewpoint, and to predict - from the graphic of Hawking temperature $\times M_{\mathrm{BH}}$ - the Planck mass and the number $n$ of extra dimensions in a more precise and realistic approach. The fact that $T_{\mathrm{H}}$ increases as the spinning parameter $a$ also increases shows that all more realistic brane-effect corrections obtained in this

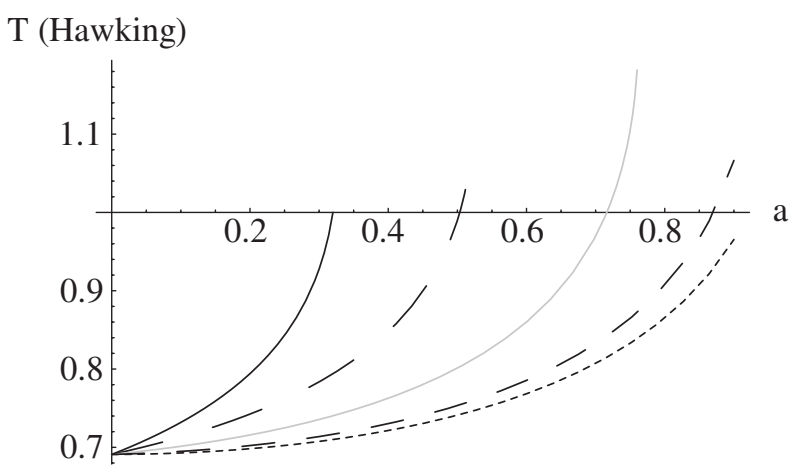

FIG. 18. Range of Hawking radiation, in $\mathrm{TeV}$, produced in LHC by a charged spinning BH evaporation versus BH spinning parameter $a$, for a fixed $\mathrm{BH}$ mass $(1 \mathrm{TeV})$ for the RandallSundrum brane-world model. Here we present an example for $M_{\mathrm{P}}=4 \mathrm{TeV}$. For the very short dashed line: $Q^{*}=-0.1$; for the short dashed line: $Q^{*}=-0.5$; for the full gray line: $Q^{*}=-1$; for the full black line: $Q^{*}=-1.5$; and for the long dashed line: $Q^{*}=-2$. In this case, the spinning of the $\mathrm{BH}$ is constrained by the electric and tidal charge.

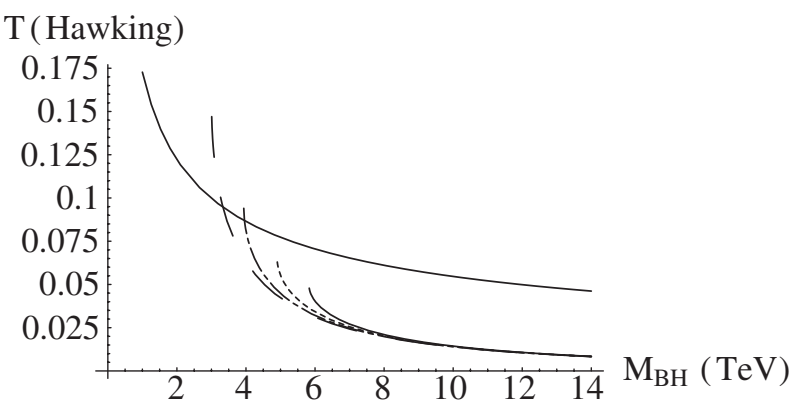

FIG. 19. Range of Hawking radiation, in $\mathrm{TeV}$, produced in LHC by a charged spinning BH evaporation versus $\mathrm{BH}$ spinning parameter $a$, for a fixed $\mathrm{BH}$ mass $(1 \mathrm{TeV})$ for the RandallSundrum brane-world model, where there is one extra warped dimension. Here we present an example for Planck mass $M_{\mathrm{P}}=$ $1 \mathrm{TeV}$. For the very short dashed line: $Q^{*}=-0.1$; for the short dashed line: $Q^{*}=-0.5$; for the full gray line: $Q^{*}=-1$; for the full black line: $Q^{*}=-1.5$; and for the long dashed line: $Q^{*}=$ -2 . In this case, the spinning of the $\mathrm{BH}$ is constrained by the electric and tidal charge.

paper for cross sections, Hawking temperature, and KerrNewman horizons, can be extremely useful in an optimal use of the range $1-10 \mathrm{TeV}$ of LHC.

The question concerning where mini-BHs mostly radiate - on the brane or on the bulk - remains open, and its answer will allow, at least in principle, to detect most of the decay products in the case of radiation on the brane. If $\mathrm{BHs}$ radiate mostly in the bulk, one has to take any constraints coming from nonobservation of such events with caution. Because of the nonzero impact parameter of initial particles, most of the produced BHs will be highly rotating, and our model described in this paper describes such processes with more accuracy. Such BHs radiate mostly in the bulk - at least as long as they rotate fast - as first pointed out in [34-36]. The reason is that rotating BHs exhibit the effect of superradiance, i.e., certain radiation modes get significantly amplified taking away rotational energy of the BH [56-59]. The model described here using KerrNewman electrically charged and rotating $\mathrm{BHs}$ gives rise to a more profound analysis of the superradiance effect, which is highly spin-dependent and radiation of higher spin particles is highly preferred-for instance gravitons are preferred to photons and neutrinos. While on a brane with $(1+3)$-dimensions there are 2 degrees of freedom of graviton, the probability of a graviton emission is a hundred times higher than the probability of a photon and neutrino emission $[60,61]$. This effect must be even stronger in the higher-dimensional case where you have more gravitational degrees of freedom available. Also, superradiance exists in higher dimensions $[58,59]$ and interactions between BHs and the brane [62-65] exist, in which the BH loses some of its components of angular momentum. Thus, after initial bulk domination, in the final stages, it is expected again that brane radiation dominates. Also, the extra-dimensional geometrical scenario is funda- 
mental for such analysis. New analysis procedures involving superradiance and charged Kerr-Newman mini-BHs are to be discussed in a forthcoming paper.

Besides, gravitational field equations on the brane can also be solved in the framework of ADM [66] formalism. For instance, in [67] a supermassive star effective gravitational field equation on the brane was recovered and it generalized the off-brane equations due to both the acceleration of the timelike 1-form fields, in the presence of general bulk energy-momentum tensor.

Finally, a mini-BH demands a sufficiently large entropy $S_{\mathrm{BH}}$ [68], where the threshold mini-BH mass is much larger, and therefore the production becomes much smaller at the LHC. For example, to require $S_{\mathrm{BH}}>25$ implies $M_{\mathrm{BH}}>5 M_{\mathrm{P}}$ and if one assumes $M_{\mathrm{P}}=2 \mathrm{TeV}$, then the threshold mini- $\mathrm{BH}$ mass is about $10 \mathrm{TeV}$, again around the limit energy in LHC.

\section{ACKNOWLEDGMENTS}

The authors are very grateful to Professor Patricio A. Letelier, Professor Dejan Stojkovic, Professor Roberto Casadio, Dr. Ricardo Antonio Mosna, and Professor Vitor Cardoso for important comments about this paper, and to Professor Alikram Aliev and Professor Ignatios Antoniadis for pointing out some missing points in a previous version of this paper. Roldão da Rocha thanks Fundação de Amparo à Pesquisa do Estado de São Paulo (FAPESP) for financial support and Carlos H. CoimbraAraújo thanks CAPES/Brasil for financial support.
[1] S. B. Giddings, S. Kachru, and J. Polchinski, Phys. Rev. D 66, 106006 (2002).

[2] L. Randall and R. Sundrum, Phys. Rev. Lett. 83, 3370 (1999).

[3] L. Randall and R. Sundrum, Phys. Rev. Lett. 83, 4690 (1999).

[4] N. Arkani-Hamed, S. Dimopoulos, and G. R. Dvali, Phys. Lett. B 429, 263 (1998).

[5] I. Antoniadis, N. Arkani-Hamed, S. Dimopoulos, and G. R. Dvali, Phys. Lett. B 436, 257 (1998).

[6] P. Horava and E. Witten, Nucl. Phys. B460, 506 (1996).

[7] A. Lukas, B. A. Ovrut, K. S. Stelle, and D. Waldram, Phys. Rev. D 59, 086001 (1999).

[8] A. Lukas, B. A. Ovrut, and D. Waldram, Phys. Rev. D 60, 086001 (1999).

[9] A. Lukas, B. A. Ovrut, and D. Waldram, Phys. Rev. D 61, 023506 (2000).

[10] C. Rovelli, Living Rev. Relativity 1, 1 (1998), as can be found in http://relativity.livingreviews.org/Articles/lrr1998-1/index.html.

[11] G. L. Alberghi, R. Casadio, D. Galli, D. Gregori, A. Tronconi, and V. Vagnoni, hep-ph/0601243.

[12] M. B. Green, J.H. Schwarz, and E. Witten, Superstring Theory (Cambridge University Press, Cambridge, England, 1987), Vols. I and II.

[13] K. R. Dienes, Phys. Rep. 287, 447 (1997).

[14] M. Kaku, Strings, Conformal Fields and M-theory (Springer-Verlag, New York, 2000).

[15] E. Kiritsis, Introduction to Superstring Theory, Leuven Notes in Mathematics and Theoretical Physics (Leuven University Press, Leuven, 1997), Vol. 9.

[16] C. Barceló and M. Visser, Nucl. Phys. B584, 415 (2000).

[17] P. K. Townsend, Nucl. Phys. B, Proc. Suppl. 58, 163 (1997).

[18] C. H. Coimbra-Araújo, R. da Rocha, and I. T. Pedron, Int. J. Mod. Phys. D 14, 1883 (2005).

[19] R. da Rocha and C.H. Coimbra-Araújo, J. Cosmol. Astropart. Phys. 12 (2005) 009.
[20] R. Gregory, R. Whisker, K. Beckwith, and C. Done, J. Cosmol. Astropart. Phys. 10 (2004) 013.

[21] M. Gogberashvili, A. S. Sakharov, and E. K. G. Sarkisyan, hep-ph/0605326.

[22] G. L. Alberghi, R. Casadio, D. Galli, D. Gregori, A. Tronconi, and V. Vagnoni, hep-ph/0601243.

[23] S. Dimopoulos and G. Landsberg, Phys. Rev. Lett. 87, 161602 (2001).

[24] R. Casadio and B. Harms, Int. J. Mod. Phys. A 17, 4635 (2002).

[25] G. Landsberg, Phys. Rev. Lett. 88, 181801 (2002).

[26] G. Landsberg, hep-ph/0205174.

[27] A. Barrau, J. Grain, and S. O. Alexeyev, Phys. Lett. B 584, 114 (2004).

[28] B. Giddings and S. Thomas, Phys. Rev. D 65, 056010 (2002).

[29] P. C. Argyres, S. Dimopoulos, and J. March-Russell, Phys. Lett. B 441, 96 (1998).

[30] R. Emparan, G. T. Horowitz, and R. C. Myers, Phys. Rev. Lett. 85, 499 (2000).

[31] T. Banks and W. Fischer, J. High Energy Phys. 06 (1999) 014.

[32] V. Cardoso, M. Cavaglia, and L. Gualtieri, Phys. Rev. Lett. 96, 071301 (2006); 96, 219902(E) (2006).

[33] V. Cardoso, M. Cavaglia, and L. Gualtieri, J. High Energy Phys. 02 (2006) 021.

[34] V. Frolov and D. Stojkovic, Phys. Rev. Lett. 89, 151302 (2002).

[35] V. Frolov and D. Stojkovic, Phys. Rev. D 66, 084002 (2002).

[36] D. Stojkovic, Phys. Rev. Lett. 94, 011603 (2005).

[37] A. N. Aliev and A.E. Gumrukcuoglu, Phys. Rev. D 71, 104027 (2005).

[38] A. N. Aliev, Mod. Phys. Lett. A 21, 751 (2006).

[39] A. N. Aliev, Phys. Rev. D 74, 024011 (2006).

[40] R. da Rocha and C.H. Coimbra-Araújo, Proc. Sci., IC2006 (2006) 065.

[41] T. Shiromizu, K. Maeda, and M. Sasaki, Phys. Rev. D 62, 
024012 (2000).

[42] R. Maartens, Living Rev. Relativity 7, 7 (2004), as can be found in http://relativity.livingreviews.org/Articles/lrr2004-7/index.html.

[43] S. Kanno and J. Soda, Gen. Relativ. Gravit. 37, 1651 (2005).

[44] R. Emparan, J. Garcia-Bellido, and N. Kaloper, J. High Energy Phys. 01 (2003) 079.

[45] R. Gregory, V. A. Rubakov, and S. M. Sibiryakov, Classical Quantum Gravity 17, 4437 (2000).

[46] N. Dadhich, R. Maartens, P. Papadopoulos, and V. Rezania, Phys. Lett. B 487, 1 (2000).

[47] D. Ida, K. Oda, and S. C. Park, Phys. Rev. D 73, 124022 (2006).

[48] R. C. Myers and M. J. Perry, Ann. Phys. (Berlin) 172, 304 (1986).

[49] J.D. Bekenstein, in Proceedings of the Eight Marcel Grossmann Meeting, Jerusalem, 1997, edited by T. Piran and R. Ruffini (World Scientific, Singapore, 1999), pp. 92-111.

[50] S. Hawking, Commun. Math. Phys. 43, 199 (1975).

[51] B. P. Dolan, J. High Energy Phys. 02 (2005) 008.

[52] G. Duffy, C. M. Harris, P. Kanti, and E. Winstanley, J. High Energy Phys. 09 (2005) 049.

[53] B. Koch, M. Bleicher, and S. Hossenfelder, J. High Energy Phys. 10 (2005) 053.
[54] A. V. Kotwal and C. Hays, Phys. Rev. D 66, 116005 (2002).

[55] M. Cavaglia, S. Das, and R. Maartens, Classical Quantum Gravity 20, L205 (2003).

[56] E. Jung, S. H. Kim, and D. K. Park, Phys. Lett. B 615, 273 (2005).

[57] J. Koga and K. Maeda, Phys. Lett. B 340, 29 (1994).

[58] V.P. Frolov and D. Stojkovic, Phys. Rev. D 68, 064011 (2003).

[59] V.P. Frolov and D. Stojkovic, Phys. Rev. D 67, 084004 (2003).

[60] D. N. Page, Phys. Rev. D 13, 198 (1976).

[61] D. N. Page, Phys. Rev. D 14, 3260 (1976).

[62] V.P. Frolov, D. V. Fursaev, and D. Stojkovic, Classical Quantum Gravity 21, 3483 (2004).

[63] V.P. Frolov, D. V. Fursaev, and D. Stojkovic, J. High Energy Phys. 06 (2004) 057.

[64] D. Stojkovic, J. High Energy Phys. 09 (2004) 061.

[65] V. P. Frolov, M. Snajdr, and D. Stojkovic, Phys. Rev. D 68, 044002 (2003).

[66] R. Arnowit, S. Deser, and C. W. Misner, in Gravitation: An Introduction to Current Research, edited by L. Witten (J. Wiley, New York, 1962).

[67] A. N. Aliev and A.E. Gumrukcuoglu, Classical Quantum Gravity 21, 5081 (2004).

[68] King-man Cheung, Phys. Rev. Lett. 88, 221602 (2002). 